\title{
The orally available Btk inhibitor ibrutinib (PCI-32765) protects against
}

osteoclast-mediated bone loss

Masahiro Shinohara $^{\text {a,b }}$, Betty Y. Chang ${ }^{\mathrm{c}}$, Joseph J. Buggy ${ }^{\mathrm{c}}$, Yusuke Nagai ${ }^{\mathrm{a}, \mathrm{d}, \mathrm{e}}$, Tatsuhiko

Kodama $^{\mathrm{f}}$, Hiroshi Asahara ${ }^{\mathrm{b}}$, Hiroshi Takayanagi ${ }^{\mathrm{a}, \mathrm{d}, \mathrm{e},{ }^{*}}$

${ }^{\mathrm{a}}$ Department of Cell Signaling,

${ }^{\mathrm{b}}$ Department of Systems BioMedicine,

Graduate School of Medical and Dental Sciences, Tokyo Medical and Dental University, Yushima 1-5-45, Bunkyo-ku, Tokyo 113-8510, Japan

${ }^{c}$ Pharmacyclics, Inc., Research Department, Sunnyvale CA, 94085-4521, USA

${ }^{\mathrm{d}}$ Department of Immunology, Graduate School of Medicine, The University of Tokyo,

${ }^{\mathrm{e} J a p a n}$ Science and Technology Agency (JST), Explorative Research for Advanced Technology (ERATO) Program, Takayanagi Osteonetwork Project,

Hongo 7-3-1, Bunkyo-ku, Tokyo 113-0033, Japan

${ }^{\mathrm{f}}$ Department of Molecular Biology and Medicine, Laboratory for Systems Biology and Medicine, Research Center for Advanced Science and Technology, The University of Tokyo, Komaba 4-6-1, Meguro-ku, Tokyo 153-8904, Japan

* Corresponding author at: Department of Immunology, Graduate School of Medicine, The University of Tokyo, Hongo 7-3-1, Bunkyo-ku, Tokyo 113-0033, Japan E-mail address: takayana@m.u-tokyo.ac.jp (H.T.)

(C) 2013. This manuscript version is made available under the Elsevier user license http://www.elsevier.com/open-access/userlicense/1.0/ 


\begin{abstract}
Bone-resorbing osteoclasts play an essential role in normal bone homeostasis, as well as in various bone disorders such as osteoporosis and rheumatoid arthritis. Previously we showed that the Tec family of tyrosine kinases is essential for the differentiation of osteoclasts and the inhibition of Btk is a promising strategy for the prevention of the bone loss in osteoclast-associated bone disorders. Here we demonstrate that an orally available Btk inhibitor, ibrutinib (PCI-32765), suppresses osteoclastic bone resorption by inhibiting both osteoclast differentiation and function. Ibrutinib downregulated the expression of NFATc1, the key transcription factor for osteoclastogenesis, and disrupted the formation of the actin ring in mature osteoclasts. In addition, genome-wide screening revealed that Btk regulates the expression of the genes involved in osteoclast differentiation and function in both an NFATc1-dependent and -independent manner. Finally, we showed that ibrutinib administration ameliorated the bone loss that developed in a RANKL-induced osteoporosis mouse model. Thus, this study suggests ibrutinib to be a promising therapeutic agent for osteoclast-associated bone diseases.
\end{abstract}

\title{
Introduction
}

Bone tissue homeostasis is tightly regulated by bone-forming osteoblasts and bone-resorbing osteoclasts $[1,2]$. While osteoblasts contribute to the formation of the bone matrix by providing the necessary bone matrix proteins and minerals, osteoclasts degrade the bone by producing proteases for matrix protein digestion and protons for the 
dissolution of the minerals in bone. An imbalance in bone formation and resorption is the underlying cause of various bone disorders, and bone loss in osteoporosis and rheumatoid arthritis (RA) mainly results from enhanced osteoclastic bone resorption [3-5]. Thus, it is an attractive therapeutic strategy to protect bone tissues by the inhibition of osteoclastic bone resorption.

Osteoclasts are multinucleated cells that attach to the bone matrix by the use of an actin ring, an actin-rich structure, and degrade bone matrix by the secretion of protons and proteases into a space that forms between the osteoclasts and the bone surface through a specialized structure known as ruffled border membranes [6]. The differentiation of osteoclasts is regulated by three signals mediated by the colony stimulating factor 1 receptor (Csf1r, also known as c-Fms or M-CSF receptor), tumor necrosis factor receptor superfamily member 11a (Tnfrsf11a, also known as receptor activator of NFkB, RANK), and certain immunoglobulin (Ig)-like receptors, including OSCAR, TREM-2, SIRP1 $\beta$ and PIR-A [2]. While M-CSF receptor signaling supports cell proliferation and the survival of the osteoclast precursor cells during osteoclastogenesis [7], the differentiation process is activated by the RANK signal in cooperation with the Ig-like receptors. The RANK signal activates NFkB and Fos, both of which are transcription factors essential for the osteoclast differentiation [2]. Ig-like receptors transmit a signal to activate phospholipase $\mathrm{C} \gamma(\mathrm{PLC} \gamma)$ through their adaptors, immunoreceptor tyrosine-based activation motif (ITAM)-harboring molecules DAP12 and $\mathrm{FcR} \gamma$ [8]. PLC $\gamma$ induces calcium oscillation, which leads to the activation of calcineurin, a $\mathrm{Ca}^{2+} /$ calmodulin-dependent phosphatase [9]. Finally, RANK and Ig-like 
receptor signals are integrated by the master transcription factor of osteoclastogenesis, nuclear factor of activated T-cells c1 (NFATc1), which induces the expression of molecules required for the bone-resorbing activity of osteoclasts, such as cathepsin $\mathrm{K}$, MMP9, the chloride channel CLC-7 and the $\mathrm{H}^{+}$-ATPase subunits [9].

The tyrosine kinases Btk and Tec are expressed in B lymphocytes and myeloid lineages and play important roles in the development and function of these lineages [10]. Previously, we demonstrated that Btk and Tec are also expressed in osteoclast lineage cells, and $\mathrm{Btk}^{-/-} \mathrm{Tec}^{-/-}$mice exhibit severe osteopetrosis due to a defect in osteoclast differentiation [11]. These kinases are activated in response to RANKL stimulation, and interact with B-cell linker (BLNK) and lymphocyte cytosolic protein 2 (Lcp2, also known as SLP-76), which are phosphorylated downstream of the Ig-like receptors. The adaptor molecules also bind PLC $\gamma$ to induce efficient phosphorylation by the kinases contained in the complexes. Thus, Btk and Tec are kinases that link RANK with the Ig-like receptors. Based on the respective phenotypes of $B t k^{-/-}$and $\mathrm{TeC}^{-/-}$mice, it is suggested that Btk has a more important role in osteoclast differentiation than Tec. In addition, the broad-based Btk inhibitor LFM-A13 had a therapeutic effect on inflammatory bone destruction and bone loss in an osteoporosis model [11]. Thus, a Btk inhibitor is a promising drug for the bone diseases associated with the increased activity of osteoclasts.

The Btk inhibitor ibrutinib binds covalently to a specific cysteine residue (Cys 481 ) in the active site of Btk so as to inhibit its enzyme activity irreversibly [12]. Oral administration of ibrutinib leads to 24 hour target inhibition supporting once daily dosing [12], suggesting that ibrutinib is a first of its kind, orally available Btk inhibitor. It has 
already been demonstrated that ibrutinib has therapeutic effects against certain types of leukemia [13] and autoimmune diseases, such as arthritis [14], in mice. Importantly, in the RA models, ibrutinib treatment protected the bone destruction in the joints by targeting B lymphocytes, macrophages and mast cells, all of which are involved in the RA pathology [14]. Although the suppressive effects of ibrutinib on osteoclasts in vitro have been shown [15], it remains unclear whether ibrutinib has a similar effect on osteoclasts in vivo. In this study, we aimed to investigate the effect of ibrutinib on osteoclast differentiation and function both in vitro and in vivo in an effort to develop a novel strategy for the treatment of osteoclast-associated bone diseases.

\section{Materials and methods}

In vitro kinase assay

In vitro kinase assay with ibrutinib was performed as described previously [12] with slight modifications. Briefly, serial dilutions of ibrutinib were incubated with various kinases in basic reaction buffer, $20 \mathrm{mM}$ Hepes ( $\mathrm{pH}$ 7.5), $10 \mathrm{mM} \mathrm{MgCl}, 1 \mathrm{mM}$ EGTA, 0.02\% Brij35, $0.02 \mathrm{mg} / \mathrm{ml} \mathrm{BSA,} 0.1 \mathrm{mM} \mathrm{Na}_{3} \mathrm{VO}_{4}, 2 \mathrm{mM}$ DTT, 1\% DMSO with poly(Glu, Tyr) as a substrate followed by the addition of ${ }^{33} \mathrm{P}-\mathrm{ATP}$ (specific activity $500 \mu \mathrm{Ci} / \mu \mathrm{l}$ ) into the reaction mixture to initiate the reaction. The kinase reaction was performed at room temperature for $120 \mathrm{~min}$.

\section{In vitro osteoclast differentiation}

In vitro osteoclast differentiation has been described previously [11]. Briefly, bone 
marrow cells were cultured with $10 \mathrm{ng} / \mathrm{ml} \mathrm{M-CSF} \mathrm{(R \& D} \mathrm{Systems)} \mathrm{for} 2$ days to obtain bone marrow-derived monocyte/macrophages (BMMs). The BMMs were cultured in an osteoclastogenic medium containing $50 \mathrm{ng} / \mathrm{ml} \mathrm{RANKL} \mathrm{(Peprotech)} \mathrm{and} 10 \mathrm{ng} / \mathrm{ml} \mathrm{M-CSF}$ in the presence of ibrutinib at the indicated concentration for a further 3 days. Osteoclastogenesis was evaluated by tartrate-resistant acid phosphatase (TRAP) staining, and TRAP-positive multinucleated cells (TRAP ${ }^{+} \mathrm{MNCs}$; more than three nuclei) were counted.

\section{In vitro osteoblast differentiation}

Cells derived from calvaria were cultured in an osteogenic medium $(50 \mu \mathrm{M}$ ascorbic acid, $10 \mathrm{nM}$ dexamethasone and $10 \mathrm{mM} \beta$-glycerophosphate) in the presence of ibrutinib and subjected to an analysis of the activity of alkaline phosphatase (after 7 days) and bone nodule formation (after 21 days), as described previously [16].

\section{Proliferation and cell survival assays}

A proliferation assay based on incorporation of 5-bromo-2'-deoxyuridine (BrdU) was used to investigate the effect of ibrutinib on osteoclast precursor cells. BMMs were cultured in the osteoclastogenic medium with or without ibrutinib for 24 hours. One hour before the collection of cells, BrdU (Roche) was added. The cells were fixed in ice-cold $70 \%$ ethanol, washed with FACS buffer, resuspended with $2 \mathrm{~N} \mathrm{HCl}$ and the residual acid neutralized with $0.1 \mathrm{M}$ sodium borate. Finally, the cells were washed, FITC-conjugated anti-BrdU antibody (BD Pharmingen) was added and the cells were analyzed by flow 
cytometry, FACS Canto II and Diva software (BD Biosciences). Apoptosis of the osteoclast precursor cells was evaluated 24 hours after RANKL stimulation using an In Situ Cell Death Detection Kit, Fluorescein (Roche), according to the manufacturer's instructions, and analyzed by flow cytometry, FACS Canto II and Diva software (BD Biosciences).

\section{Pit formation assay}

Bone marrow cells were cultured on RepCell ${ }^{\circledR}$ (CellSeed Inc.) to generate mature osteoclasts as described above, and an equal number of osteoclasts were transferred onto dentin slices. Two days after the culture with ibrutinib at various concentrations in the presence of M-CSF and RANKL, the dentin slices were stained with $0.5 \%$ toluidine blue.

The resorption pit area was then measured, as described previously [17]. The effect of ibrutinib on the apoptosis of the mature osteoclasts was analyzed with cells transferred on a 24-well plate, and cultured in the presence of M-CSF and RANKL with or without ibrutinib for 2 days. The number of TUNEL-positive apoptotic cells labeled with In Situ Cell Death Detection Kit Fluorescein (Roche) was counted using a fluorescein microscopy.

\section{GeneChip analysis}

BMMs were cultured with RANKL and M-CSF in the presence of $10 \mathrm{nM}$ ibrutinib for 3 days. Affymetrix GeneChip analysis was performed as previously described [11]. The preparation of NFATc1-deficient [16] and DAP12/FcR $\gamma$-deficient cells [8] is described 
elsewhere.

\section{Real-time PCR analysis}

BMMs or differentiated osteoclasts were cultured with RANKL/M-CSF in the presence of ibrutinib for 3 days (Fig. 2) or 24 hours (Fig. 3), respectively. Real-time PCR was performed using specific primers for Nfatc1, Acp5 and Ctsk as described previously [17].

\section{Western blot analysis}

For the detection of tyrosine phosphorylation of PLC $\gamma 1$ and PLC $\gamma 2$, the BMMs were treated with RANKL for 24 hours and then serum-starved for 4 hours with or without ibrutinib. The cells were treated with RANKL (50 ng/ml) for 10 minutes. Detection of the tyrosine phosphorylation of PLC $\gamma 1$ and PLC $\gamma 2$ was performed as described previously [11]. The expression of Src was detected with an anti-Src antibody (Cell Signaling).

$\mathrm{Ca}^{2+}$ measurement

BMMs were cultured with RANKL and M-CSF for 48 hours with or without ibrutinib (10 $\mathrm{nM}$ ). The concentration of intracellular calcium was measured as described previously [11].

\section{Analysis of actin structure}

Osteoclasts cultured on dentin slices were treated with $10 \mathrm{nM}$ ibrutinib for 8 hours. Cells 
were fixed with PBS containing 4\% paraformaldehyde, permeabilized with PBS containing $0.2 \%$ Triton-X100, and treated with Rhodamine-labeled phalloidin (Molecular Probes). The images were taken using a confocal laser scanning microscopy Leica TSC SP5 (Leica Microsystems) as described previously [17].

RANKL-induced bone loss in mice

GST-RANKL recombinant protein was purified from BL21 cells as previously described [18]. After purification using glutathione-Sepharose 4B and Mono-Q column (GE Healthcare), the protein was further purified with the ToxinEraser ${ }^{\mathrm{TM}}$ Endotoxin Removal Kit (GenScript). We confirmed the endotoxin level to be less than $50 \mathrm{EU} / \mathrm{mg}$ protein. Female C57BL/6JJc1 mice, aged 8 weeks, were intraperitoneally injected with $0.5 \mathrm{mg} / \mathrm{kg}$ of GST-RANKL three times at 24-hour intervals. Ibrutinib was administered by oral gavage 1 hour before the first GST-RANKL injection. One and one-half hours after the last injection, all of the mice were sacrificed and subjected to microcomputed tomography (microCT) and bone histomorphometrical analyses. MicroCT and bone histomorphometrical analyses were performed as described previously [17]. All animal experiments were performed with the approval of the Animal Study Committee of Tokyo Medical and Dental University and conformed to all relevant guidelines and laws.

\section{Statistical analysis}

All experiments were performed at least three times. Statistical analysis was performed using Student's $t$-test $\left({ }^{*} P<0.05,{ }^{* *} P<0.01,{ }^{* * *} P<0.005\right.$, n.s.: not significant, throughout 
the paper). In Figure 4, significant differences are indicated with asterisks (saline versus the RANKL-treated group; ${ }^{*} P<0.05,{ }^{* *} P<0.01,{ }^{* * *} P<0.005$, n.s.: not significant) and hash marks (RANKL-treated versus the RANKL- and ibrutinib-treated group; ${ }^{\#} P<0.05$, ${ }^{\# \#} P<0.01,{ }^{\# \#} P<0.005$, n.s.: not significant).

\section{Results}

Ibrutinib inhibits osteoclast differentiation

We previously reported that ibrutinib (PCI-32765) is a potent inhibitor of Btk [12]. To determine the biochemical activity of ibrutinib, we performed an in vitro kinase assay using various kinases with serial dilutions of ibrutinib. Since the drug is a covalent inhibitor with a high systemic clearance, only those kinases with a high homology to Btk and containing a cysteine residue aligned with Cys 481 in Btk, such as other Tec family kinases (Bmx, Txk and Tec), B lymphocyte kinase (Blk), and ErbB4/HER4 were inhibited, thus affording selectivity over the kinases that are inhibited reversibly (Table S1).

We evaluated the effect of the ibrutinib on osteoclast differentiation in a culture system of bone marrow-derived monocyte/macrophages (BMMs) stimulated with RANKL and M-CSF. The formation of tartrate-resistant acid phosphatase (TRAP)-positive osteoclasts was potently suppressed by ibrutinib in a dose-dependent manner (Figs. 1A, B) in the same dose range as blocked the activation of B lymphocytes. While TRAP-positive mononuclear cells were observed at 3 and $10 \mathrm{nM}$, no TRAP-positive cells were detected at higher doses (Fig. 1A). We also analyzed the cell 
proliferation and survival of osteoclast precursor cells by BrdU incorporation and TUNEL assays, respectively, and there was no significant difference between the control and ibrutinib-treated cells (Figs. 1C, D), suggesting that ibrutinib affects osteoclast differentiation by inhibiting RANKL but not M-CSF signaling. In contrast, the differentiation of osteoblasts was not affected by ibrutinib at concentrations that inhibit osteoclastogenesis (Figs. 1E, F, G). These results are consistent with the previous observations showing that osteoclastogenesis, but not osteoblastogenesis, was impaired in Btk-deficient cells [11], suggesting that ibrutinib specifically inhibits bone resorption without affecting bone formation.

\section{Suppression of osteoclast gene expression by ibrutinib}

We next analyzed the signaling events stimulated by Btk in response to RANKL. The tyrosine phosphorylation of PLC $\gamma 1$ and $\gamma 2$ induced by RANKL stimulation was attenuated by the treatment with ibrutinib dose-dependently (Fig. 2A). Furthermore, the calcium oscillation observed during osteoclast differentiation was completely blocked by $10 \mathrm{nM}$ ibrutinib treatment (Fig. 2B). The expression of NFATc1, the master transcription factor of osteoclast differentiation [9], was increasingly suppressed by increasing doses of ibrutinib (Fig. 2C). Consistent with this, real-time PCR analysis revealed that the expression of the osteoclast-specific genes Acp5 encoding TRAP and Ctsk was decreased by the treatment with ibrutinib (Fig. 2D). We also examined the expression of RANKL-induced genes in osteoclast differentiation using a genome-wide approach. Affymetrix GeneChip analysis revealed that the genes thought to be important 
for osteoclast differentiation and function, such as Ppargc1b [19], Fosl2 [20], Nfkb2 [21], Atp6v1h encoding a subunit of the proton pump [22], Clcn7 [23], Src [24] and Itgb3 [25], were suppressed by ibrutinib (Fig. 2E). These results suggest that ibrutinib blocks osteoclast differentiation through the inhibition of NFATc1 expression and thus the subsequent expression of osteoclast-related genes.

Ibrutinib inhibits bone-resorbing activity of osteoclasts

Src tyrosine kinase is essential for proper osteoclastic bone resorption [24] and previous data suggests that Src expression is dramatically induced after RANKL stimulation [11]. Consistent with this, our GeneChip data showed that Src expression was induced during osteoclast differentiation and that this induction was suppressed by ibrutinib treatment (Fig. 3A). The suppression of Src expression was confirmed by Western blot analysis (Fig. 3B). Interestingly, the expression of Src was normally induced in NFATc1- as well as DAP12/FcR $\gamma$-deficient cells 3 days after RANKL stimulation of BMMs (Fig. 3C), suggesting that ibrutinib suppressed Src expression in an NFATc1-independent fashion. These results led us to analyze the effect of ibrutinib on the bone-resorbing activity of osteoclasts. Pit formation assay revealed that ibrutinib inhibited osteoclastic bone-resorbing activity partially at $10 \mathrm{nM}$ but completely at 100 nM (Fig. 3D). Since the rate of apoptosis was not changed by the ibrutinib treatment (Fig. 3D), ibrutinib suppressed the bone-resorbing activity without affecting cell survival. In this condition, Src expression was decreased by ibrutinib treatment (Fig. 3E). Furthermore, we analyzed the expression of genes related to bone resorption, and found 
that protein tyrosine kinase 2 (Ptk2; also known as Focal adhesion kinase, FAK), Ptk2b (also known as Pyk2) and Talin $1(\mathrm{~T} \ln 1)$, which have important roles in the regulation of the actin ring in cooperation with integrins and Src [26, 27], were significantly suppressed by ibrutinib (Figs. 3F, G). In accordance with these observations, the actin ring essentially disappeared within 8 hours after ibrutinib treatment (Fig. 3H), suggesting that the suppression of bone-resorbing activity by ibrutinib is due to the disruption of the actin ring.

Ibrutinib protects against bone loss in a RANKL-induced osteoporosis model

We investigated whether ibrutinib would exert in vivo effects in a mouse model of osteoporosis associated with enhanced osteoclastic bone resorption [18]. Eight-week-old female mice were injected with GST-RANKL at a 24-hour interval for 3 days. Ibrutinib was administered with a single oral treatment 1 hour before the initial RANKL injection. Three-dimensional images of the distal region of the femur showed a robust trabecular bone loss, and microCT analysis revealed a marked reduction in bone volume, thickness and trabecular number, along with an increase in trabecular separation in the RANKL-injected mice (Fig. 4A). The treatment with ibrutinib protected against the trabecular bone loss induced by the RANKL injection (Fig. 4A). The bone volume, trabecular thickness and trabecular number were increased, and trabecular separation was decreased by ibrutinib administration (Fig. 4A). Furthermore, histological analysis revealed that the loss of trabecular bone induced by RANKL was cancelled by ibrutinib injection (Fig. 4B). Increased osteoclast parameters, such as the eroded bone surface, 
osteoclast surface, and osteoclast number after RANKL injection, were suppressed dose-dependently by ibrutinib treatment (Fig. 4B). Serum level of calcium and C-telopeptide of type 1 collagen (CTX), which was enhanced by RANKL injection, was inhibited dose-dependently (Fig. 4C), suggesting enhanced bone resorption was suppressed by the ibrutinib treatment. Serum alkaline phosphatase (ALP) level was also elevated by RANKL injection as reported previously [18] but there was no effect of the ibrutinib treatment on the serum ALP level. In addition, serum phosphate level was not changed in this model (Fig. 4C). Taken together, these results suggest that ibrutinib ameliorates the bone loss induced by enhanced osteoclast activity by a direct effect on osteoclasts.

\section{Discussion}

Previously we demonstrated that the tyrosine kinases Btk and Tec are essential for the osteoclast differentiation, and the Btk inhibitor LFM-A13 blocks osteoclast formation both in vitro and in vivo [11]. LFM-A13 was originally developed to block the kinase activity of Btk by binding to the catalytic site in its kinase domain [28], and exhibited Btk inhibitory activity along with Tec inhibitory activity [29]. However, since LFM-A13 is not orally available and has high cell toxicity, its usage has been limited to in vitro experiments. The in vitro osteoclast differentiation was significantly suppressed, with an $\mathrm{IC}_{50}$ of approximately $30 \mu \mathrm{M}$, and was completely blocked at $100 \mu \mathrm{M}$. In contrast, we observed that ibrutinib completely inhibited the in vitro osteoclast differentiation at $1 \mathrm{nM}$. Furthermore, ibrutinib has an advantage over LFM-A13 in terms 
of toxicity. The mice treated with LFM-A13 at the effective dose often were debilitated and subsequently died (data not shown), but such adverse events were not observed in the mice treated with ibrutinib. Since LFM-A13 inhibits not only Tec kinases but also Janus kinases (JAKs) [30] and Polo-like kinases (PLKs) [31], which might be the reason for the unexpected side effects in mice. In contrast, ibrutinib treatment did not affect serum levels of a variety of components determined by blood chemistry tests in a various mouse model [14], and there was no effect of ibrutinib on liver and renal functions even in humans [32-34], suggesting that ibrutinib has no severe side effects. Collectively, the usage of ibrutinib in the clinical treatment of osteoclast-associated bone diseases warrants further investigation.

The master transcription factor NFATc1 is essential for the expression of osteoclast-related genes [4]. The initial induction of this transcription factor is largely dependent on Fos and NFkB, which are activated by RANKL stimulation in the early phase of osteoclast differentiation, and subsequently activated through dephosphorylation by calcineurin, a protein phosphatase regulated by calcium signaling [9]. The promoter region in the Nfatc1 gene contains NFAT-binding sequences as well as the Fos- and NFKB-binding sites, which enable the autoamplification of NFATc1 in order to maintain the high expression level that is required during the course of osteoclastogenesis [35]. Concomitant with the autoamplification of NFATc1, it has been shown that NFATc1 also activates the gene expression of various molecules associated with osteoclast differentiation and function, such as TRAP, OSCAR, cathepsin K, the chloride channel and the proton pump subunits. The data show that calcium oscillation 
and NFATc1 expression were significantly suppressed in the cells treated with ibrutinib, resulting in the inhibition of the osteoclast differentiation through the down-regulation of a wide range of NFATc1 targets.

Our results also suggest that Btk regulates gene expression independently of NFATc1 in osteoclasts. Src is evidently essential for osteoclastic bone resorption, because mice deficient in Src exhibit severe osteopetrosis due to a lack of the bone-resorbing activity of osteoclasts [24]. However, the mechanism of Src expression in osteoclasts has long been largely unknown. Our data indicate that Src expression was not suppressed in NFATc1-deficient cells. In addition, the expression of Ptk2, Ptk2b and Tln1, was also expressed in NFATc1-deficient cells (Fig. S1), suggesting that the expression of these genes is independent of NFATc1 but dependent on Btk. In contrast, it is well known that expression of CtsK and Acp5 is under the control of NFATc1 [9, 36]. Since ibrutinib inhibited expression of both NFATc1-dependent (CtsK and Acp5) and -independent (Src, Ptk2, Ptk2b and Tln1) genes, Btk regulates the gene expression in the NFATc1-dependent and -independent manner in osteoclast linage cells. Previously it has been shown that Btk controls gene expression by regulating activity of transcription factors NFkB [37], AT rich interactive domain 3A (Arid3a, also known as Bright) [38], and signal transducer and activator of transcription 5 (Stat5) [39]. Since these transcription factors are expressed in the osteoclast lineage (data not shown), it is conceivable that Btk regulates gene expression through these factors in osteoclasts as well.

For the treatment of osteoclast-associated bone diseases, such as osteoporosis and RA, the therapeutic regulation of osteoclastic bone resorption is critically important 
[9]. Bisphosphonates, which inhibit the survival and the bone-resorbing activity of osteoclasts, are widely used for osteoporosis treatment [40]. More recently, an anti-RANKL antibody was developed and shown to be an effective therapeutic agent for the treatment of osteoporosis [41]. It also has been shown that biological agents against TNF [42] and IL-6 [43], which are commonly used for RA treatment, effectively prevent the bone destruction in RA by a direct or indirect inhibition of osteoclast differentiation and function, as well as the suppression of immune cell function. However, considering the side effects and cost, the development of novel drugs for the treatment of osteoclast-associated bone diseases is still greatly desired. A previous study revealed that ibrutinib ameliorates the inflammation and subsequent bone destruction which occurs in RA mouse models, suggesting that ibrutinib is a promising drug for RA [14]. Taken together with our results, it is clear that ibrutinib suppresses bone destruction by targeting both immune cells and osteoclasts. Furthermore, the direct effect of ibrutinib on osteoclasts also suggests a potential application to osteoporosis. Although further detailed investigation is required, the administration of ibrutinib is suggested to be an interesting new strategy for the treatment of osteoclast-mediated bone diseases.

\section{Conclusion}

It was demonstrated that ibrutinib inhibits osteoclast differentiation and function in vitro by regulating the expression of osteoclast-associated genes. Furthermore, this study showed that oral administration of ibrutinib protects against bone loss in a mouse model of osteoporosis, suggesting that this Btk inhibitor is a potential 
therapeutic agent for certain osteoclast-related diseases, such as osteoporosis and RA.

\section{Conflict of interest statement}

B.Y.C. and J.J.B. are employees of Pharmacyclics Inc. and have a financial interest in ibrutinib. H.T. has received research support from Pharmacyclics Inc. The remaining authors declare no competing financial interests.

\section{Acknowledgements}

We thank A. Ishizaki-Yamamura, T. Oishi-Ando, Y. Ogiwara, H. Otsuka and T. Negishi-Koga for discussion and technical assistance. This work was supported in part by Pharmacyclics Inc. and a grant for ERATO, the Takayanagi Osteonetwork Project from the Japan Science and Technology Agency (H.T.); Grant-in-Aid for Young Scientist A and Challenging Exploratory Research from the Japan Society for the Promotion of Science, and grants from Kowa Life Science Foundation, Takeda Science Foundation and Kanae Foundation for the Promotion of Medical Science (M.S.).

\section{References}

[1] Karsenty G, Wagner EF. Reaching a genetic and molecular understanding of skeletal development. Dev Cell 2002;2: 389-406.

[2] Takayanagi H. Osteoimmunology: shared mechanisms and crosstalk between the immune and bone systems. Nat Rev Immunol 2007;7: 292-304.

[3] Rodan GA, Martin TJ. Therapeutic approaches to bone diseases. Science 
2000;289: 1508-14.

[4] Takayanagi H. Osteoimmunology and the effects of the immune system on bone. Nat Rev Rheumatol 2009;5: 667-76.

[5] Theill LE, Boyle WJ, Penninger JM. RANK-L and RANK: T cells, bone loss, and mammalian evolution. Annu Rev Immunol 2002;20: 795-823.

[6] Teitelbaum SL, Ross FP. Genetic regulation of osteoclast development and function. Nat Rev Genet 2003;4: 638-49.

[7] Ross FP, Teitelbaum SL. $\alpha_{\mathrm{v}} \beta_{3}$ and macrophage colony-stimulating factor: partners in osteoclast biology. Immunol Rev 2005;208: 88-105.

[8] Koga T, Inui M, Inoue K, Kim S, Suematsu A, Kobayashi E, Iwata T, Ohnishi H, Matozaki T, Kodama T, Taniguchi T, Takayanagi H, Takai T. Costimulatory signals mediated by the ITAM motif cooperate with RANKL for bone homeostasis. Nature 2004;428: 758-63.

[9] Takayanagi H, Kim S, Koga T, Nishina H, Isshiki M, Yoshida H, Saiura A, Isobe M, Yokochi T, Inoue J, Wagner EF, Mak TW, Kodama T, Taniguchi T. Induction and activation of the transcription factor NFATc1 (NFAT2) integrate RANKL signaling in terminal differentiation of osteoclasts. Dev Cell 2002;3: 889-901.

[10] Schmidt U, Boucheron N, Unger B, Ellmeier W. The role of Tec family kinases in myeloid cells. Int Arch Allergy Immunol 2004;134: 65-78.

[11] Shinohara M, Koga T, Okamoto K, Sakaguchi S, Arai K, Yasuda H, Takai T, Kodama T, Morio T, Geha RS, Kitamura D, Kurosaki T, Ellmeier W, Takayanagi H. Tyrosine kinases Btk and Tec regulate osteoclast differentiation by linking RANK and 
ITAM signals. Cell 2008;132: 794-806.

[12] Honigberg LA, Smith AM, Sirisawad M, Verner E, Loury D, Chang B, Li S, Pan Z, Thamm DH, Miller RA, Buggy JJ. The Bruton tyrosine kinase inhibitor PCI-32765 blocks B-cell activation and is efficacious in models of autoimmune disease and B-cell malignancy. Proc Natl Acad Sci U S A 2010;107: 13075-80.

[13] Herman SE, Gordon AL, Hertlein E, Ramanunni A, Zhang X, Jaglowski S, Flynn J, Jones J, Blum KA, Buggy JJ, Hamdy A, Johnson AJ, Byrd JC. Bruton tyrosine kinase represents a promising therapeutic target for treatment of chronic lymphocytic leukemia and is effectively targeted by PCI-32765. Blood 2011;117: 6287-96.

[14] Chang BY, Huang MM, Francesco M, Chen J, Sokolove J, Magadala P, Robinson WH, Buggy JJ. The Bruton tyrosine kinase inhibitor PCI-32765 ameliorates autoimmune arthritis by inhibition of multiple effector cells. Arthritis Res Ther 2011;13: R115.

[15] Tai YT, Chang BY, Kong SY, Fulciniti M, Yang G, Calle Y, Hu Y, Lin J, Zhao JJ, Cagnetta A, Cea M, Sellitto MA, Zhong MY, Wang Q, Acharya C, Carrasco DR, Buggy JJ, Elias L, Treon SP, Matsui W, Richardson P, Munshi NC, Anderson KC. Bruton tyrosine kinase inhibition is a novel therapeutic strategy targeting tumor in the bone marrow microenvironment in multiple myeloma. Blood 2012;120: 1877-87.

[16] Negishi-Koga T, Shinohara M, Komatsu N, Bito H, Kodama T, Friedel RH, Takayanagi H. Suppression of bone formation by osteoclastic expression of semaphorin 4D. Nat Med 2011;17: 1473-80.

[17] Shinohara M, Nakamura M, Masuda H, Hirose J, Kadono Y, Iwasawa M, 
Nagase Y, Ueki K, Kadowaki T, Sasaki T, Kato S, Nakamura H, Tanaka S, Takayanagi H. Class IA phosphatidylinositol 3-kinase regulates osteoclastic bone resorption through protein kinase B-mediated vesicle transport. J Bone Miner Res 2012;27: 2464-75.

[18] Tomimori Y, Mori K, Koide M, Nakamichi Y, Ninomiya T, Udagawa N, Yasuda H. Evaluation of pharmaceuticals with a novel 50-hour animal model of bone loss. J Bone Miner Res 2009;24: 1194-205.

[19] Ishii KA, Fumoto T, Iwai K, Takeshita S, Ito M, Shimohata N, Aburatani H, Taketani S, Lelliott CJ, Vidal-Puig A, Ikeda K. Coordination of PGC-1 $\beta$ and iron uptake in mitochondrial biogenesis and osteoclast activation. Nat Med 2009;15: 259-66.

[20] Bozec A, Bakiri L, Hoebertz A, Eferl R, Schilling AF, Komnenovic V, Scheuch H, Priemel M, Stewart CL, Amling M, Wagner EF. Osteoclast size is controlled by Fra-2 through LIF/LIF-receptor signalling and hypoxia. Nature 2008;454: 221-5.

[21] Novack DV, Yin L, Hagen-Stapleton A, Schreiber RD, Goeddel DV, Ross FP,

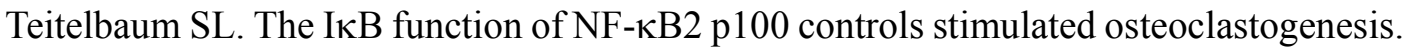
J Exp Med 2003;198: 771-81.

[22] Sun-Wada GH, Wada Y, Futai M. Diverse and essential roles of mammalian vacuolar-type proton pump ATPase: toward the physiological understanding of inside acidic compartments. Biochim Biophys Acta 2004;1658: 106-14.

[23] Kornak U, Kasper D, Bosl MR, Kaiser E, Schweizer M, Schulz A, Friedrich W, Delling G, Jentsch TJ. Loss of the ClC-7 chloride channel leads to osteopetrosis in mice and man. Cell 2001;104: 205-15.

[24] Soriano P, Montgomery C, Geske R, Bradley A. Targeted disruption of the c-src 
proto-oncogene leads to osteopetrosis in mice. Cell 1991;64: 693-702.

[25] McHugh KP, Hodivala-Dilke K, Zheng MH, Namba N, Lam J, Novack D, Feng

X, Ross FP, Hynes RO, Teitelbaum SL. Mice lacking $\beta 3$ integrins are osteosclerotic because of dysfunctional osteoclasts. J Clin Invest 2000;105: 433-40.

[26] Horne WC, Sanjay A, Bruzzaniti A, Baron R. The role(s) of Src kinase and Cbl proteins in the regulation of osteoclast differentiation and function. Immunol Rev 2005;208: 106-25.

[27] Zou W, Izawa T, Zhu T, Chappel J, Otero K, Monkley SJ, Critchley DR, Petrich BG, Morozov A, Ginsberg MH, Teitelbaum SL. Talin1 and Rap1 are critical for osteoclast function. Mol Cell Biol 2013;33: 830-44.

[28] Mahajan S, Ghosh S, Sudbeck EA, Zheng Y, Downs S, Hupke M, Uckun FM. Rational design and synthesis of a novel anti-leukemic agent targeting Bruton's tyrosine kinase (BTK), LFM-A13 [ $\quad$-cyano- $\beta$-hydroxy- $\beta$-methyl-N-(2, 5-dibromophenyl)propenamide]. J Biol Chem 1999;274: 9587-99.

[29] Fernandes MJ, Lachance G, Pare G, Rollet-Labelle E, Naccache PH. Signaling through CD16b in human neutrophils involves the Tec family of tyrosine kinases. J Leukoc Biol 2005;78: 524-32.

[30] van den Akker E, van Dijk TB, Schmidt U, Felida L, Beug H, Lowenberg B, von Lindern M. The Btk inhibitor LFM-A13 is a potent inhibitor of Jak2 kinase activity. Biol Chem 2004;385: 409-13.

[31] Uckun FM, Dibirdik I, Qazi S, Vassilev A, Ma H, Mao C, Benyumov A, Emami KH. Anti-breast cancer activity of LFM-A13, a potent inhibitor of Polo-like kinase (PLK). 
Bioorg Med Chem 2007;15: 800-14.

[32] Advani RH, Buggy JJ, Sharman JP, Smith SM, Boyd TE, Grant B, Kolibaba KS, Furman RR, Rodriguez S, Chang BY, Sukbuntherng J, Izumi R, Hamdy A, Hedrick E, Fowler NH. Bruton tyrosine kinase inhibitor ibrutinib (PCI-32765) has significant activity in patients with relapsed/refractory B-cell malignancies. J Clin Oncol 2013;31: $88-94$.

[33] Byrd JC, Furman RR, Coutre SE, Flinn IW, Burger JA, Blum KA, Grant B, Sharman JP, Coleman M, Wierda WG, Jones JA, Zhao W, Heerema NA, Johnson AJ, Sukbuntherng J, Chang BY, Clow F, Hedrick E, Buggy JJ, James DF, O'Brien S. Targeting BTK with ibrutinib in relapsed chronic lymphocytic leukemia. N Engl J Med 2013;369: 32-42.

[34] Wang ML, Rule S, Martin P, Goy A, Auer R, Kahl BS, Jurczak W, Advani RH, Romaguera JE, Williams ME, Barrientos JC, Chmielowska E, Radford J, Stilgenbauer S, Dreyling M, Jedrzejczak WW, Johnson P, Spurgeon SE, Li L, Zhang L, Newberry K, Ou Z, Cheng N, Fang B, McGreivy J, Clow F, Buggy JJ, Chang BY, Beaupre DM, Kunkel LA, Blum KA. Targeting BTK with ibrutinib in relapsed or refractory mantle-cell lymphoma. N Engl J Med 2013;369: 507-16.

[35] Asagiri M, Sato K, Usami T, Ochi S, Nishina H, Yoshida H, Morita I, Wagner EF, Mak TW, Serfling E, Takayanagi H. Autoamplification of NFATc1 expression determines its essential role in bone homeostasis. J Exp Med 2005;202: 1261-9.

[36] Matsumoto M, Kogawa M, Wada S, Takayanagi H, Tsujimoto M, Katayama S, Hisatake K, Nogi Y. Essential role of p38 mitogen-activated protein kinase in cathepsin K 
gene expression during osteoclastogenesis through association of NFATc1 and PU.1. J Biol Chem 2004;279: 45969-79.

[37] Petro JB, Rahman SM, Ballard DW, Khan WN. Bruton's tyrosine kinase is required for activation of $\mathrm{I} \kappa \mathrm{B}$ kinase and nuclear factor $\kappa \mathrm{B}$ in response to $\mathrm{B}$ cell receptor engagement. J Exp Med 2000;191: 1745-54.

[38] Webb CF, Yamashita Y, Ayers N, Evetts S, Paulin Y, Conley ME, Smith EA. The transcription factor Bright associates with Bruton's tyrosine kinase, the defective protein in immunodeficiency disease. J Immunol 2000;165: 6956-65.

[39] Mahajan S, Vassilev A, Sun N, Ozer Z, Mao C, Uckun FM. Transcription factor STAT5A is a substrate of Bruton's tyrosine kinase in B cells. J Biol Chem 2001;276: 31216-28.

[40] Baron R, Ferrari S, Russell RG. Denosumab and bisphosphonates: different mechanisms of action and effects. Bone 2011;48: 677-92.

[41] Lacey DL, Boyle WJ, Simonet WS, Kostenuik PJ, Dougall WC, Sullivan JK, San Martin J, Dansey R. Bench to bedside: elucidation of the OPG-RANK-RANKL pathway and the development of denosumab. Nat Rev Drug Discov 2012;11: 401-19.

[42] Taylor PC. How do the efficacy and safety of abatacept and infliximab compare in the treatment of active RA? Nat Clin Pract Rheumatol 2009;5: 126-7.

[43] Tanaka T, Narazaki M, Kishimoto T. Therapeutic targeting of the interleukin-6 receptor. Annu Rev Pharmacol Toxicol 2012;52: 199-219. 


\section{Figure legends}

Figure 1. Ibrutinib inhibits the differentiation of osteoclasts, but not osteoblasts, in vitro.

A, Representative images of cells cultured in the presence of ibrutinib at the indicated concentration during osteoclast differentiation. B, The number of TRAP-positive mature osteoclasts in Fig. 1A. Data are represented as the mean \pm SEM of 3 independent experiments. C, Proliferation rate of ibrutinib-treated BMMs after RANKL stimulation for 48 hours. D, Survival rate of the ibrutinib-treated BMMs after RANKL stimulation for 48 hours. UV-treated cells were analyzed as a positive control. E, Representative images of alkaline phosphatase (upper) and alizarin red (lower) staining. F, Effect of ibrutinib on alkaline phosphatase activity. G, Quantification of the amount of alizarin red in Fig. 1E.

Figure 2. Ibrutinib suppresses the expression of osteoclast-related genes.

A, Tyrosine phosphorylation of PLC $\gamma$ after RANKL stimulation of osteoclast precursor

cells. B, Intracellular $\mathrm{Ca}^{2+}$ concentration in the control (left) and ibrutinib (10 nM)-treated (right) BMMs 2 days after stimulation with RANKL. C, Expression level of Nfatc1 in ibrutinib-treated BMMs 3 days after RANKL stimulation. D, Expression level of Acp5 (left) and Ctsk (right) in ibrutinib-treated BMMs 3 days after RANKL stimulation. E, Expression profile of the osteoclast-related genes in the ibrutinib-treated BMMs 3 days after RANKL stimulation. determined by GeneChip analysis. The relative expression level in the ibrutinib-treated cells compared with untreated cells is shown. 
Figure 3. Ibrutinib inhibits bone-resorbing activity through the disruption of actin ring. A, Src expression in control and ibrutinib-treated cells after RANKL stimulation as determined by GeneChip analysis. B, Src expression in BMMs cultured with RANKL and M-CSF for 3 days in the presence of ibrutinib at indicated concentration. C, SrC expression in control, ibrutinib-treated (Ibrutinib), NFATc1-deficient (NFATc1 KO), and DAP12/FcR $\gamma$-deficient (ITAM DKO) BMMs 3 days after culture with RANKL as determined by GeneChip analysis. D, Bone-resorbing activity of osteoclasts treated with ibrutinib. Mature osteoclasts cultured on dentin slices were treated with ibrutinib at indicated concentrations for 2 days in the presence of RANKL and M-CSF. The graphs indicate the relative amount of the resorbed area (left) and the ratio of apoptotic cells determined by TUNEL assay (right). E, Src expression in osteoclasts treated with ibrutinib at indicated concentrations for 2 days. F, Expression level of Ptk2 and Ptk2b in control and ibrutinib-treated BMMs 72 hours after RANKL stimulation as determined by GeneChip analysis. G, Tln1 expression in control and ibrutinib-treated BMMs 72 hours after RANKL stimulation as determined by GeneChip analysis. H, Actin structure in the osteoclasts treated with or without ibrutinib $(10 \mathrm{nM})$. The nuclei were shown by Hoechst staining. The scale bars indicate $10 \mu \mathrm{m}$. Right graph indicates the number of cells with actin ring.

Figure 4. Oral administration of ibrutinib protects against RANKL-induced bone loss in mice.

A, Microcomputed tomography (microCT) analysis of the femur in mice treated with 
saline, GST-RANKL or GST-RANKL along with $6.25,12.5$ or $25 \mathrm{mg} / \mathrm{kg}$ ibrutinib (upper photograph: longitudinal view; lower photograph: axial view of the metaphyseal region). The graphs indicate the parameters of the metaphyseal region on microCT analysis. $\mathrm{B}$, Histomorphometric analysis of the tibia in mice treated with saline, GST-RANKL, or GST-RANKL along with $6.25,12.5$ or $25 \mathrm{mg} / \mathrm{kg}$ ibrutinib. C, Serum levels of calcium (Ca), C-telopeptide of type 1 collagen (CTX), alkaline phosphatase (ALP) and phosphate (Pi). Data are represented as the mean \pm SEM of 9 mice. The asterisks and hash marks show the significant differences between the saline group versus the RANKL+saline group, and the RANKL+saline group versus the RANKL+ibrutinib group, respectively. 
Figure 1

A
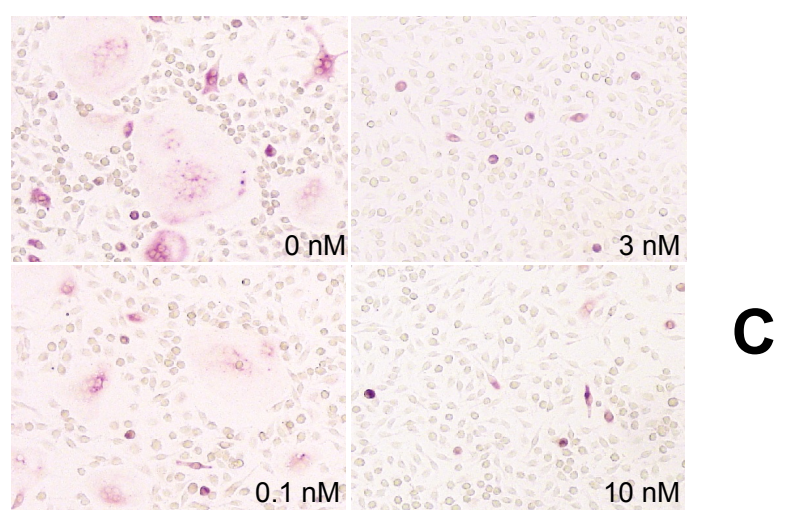

$1 \mathrm{nM}$

B

C

$30 \mathrm{nM}$

D
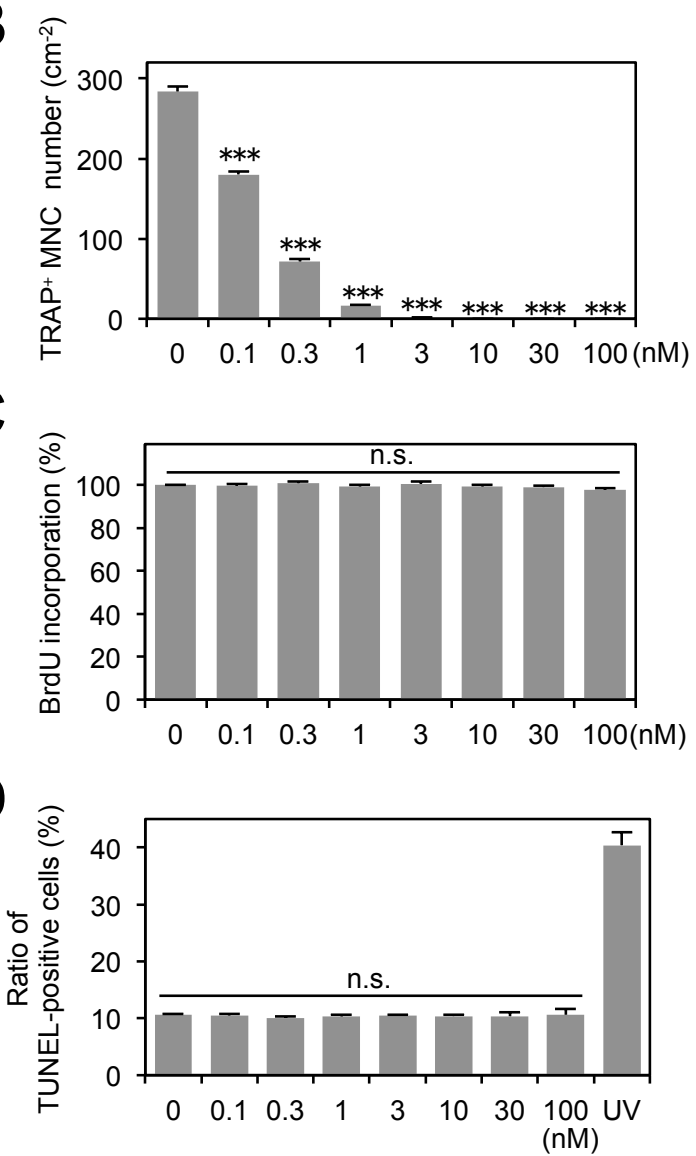

E

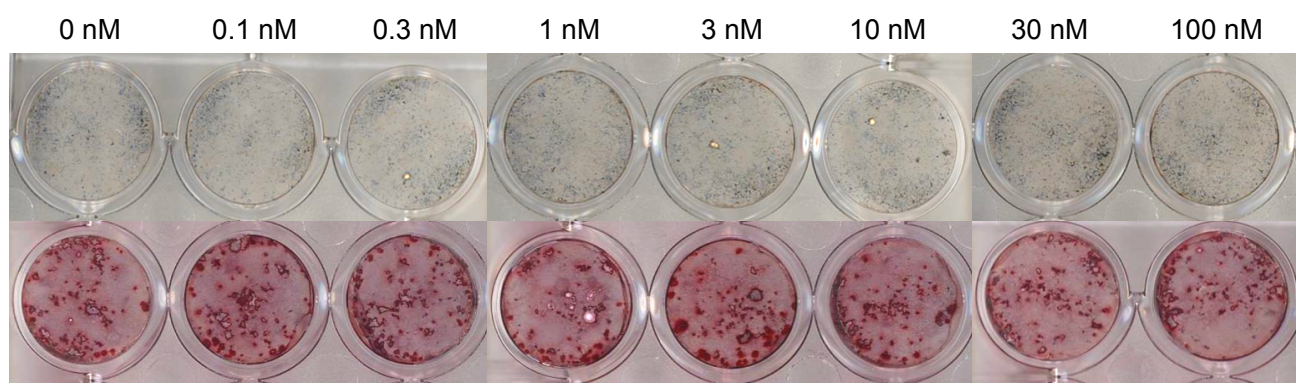

F

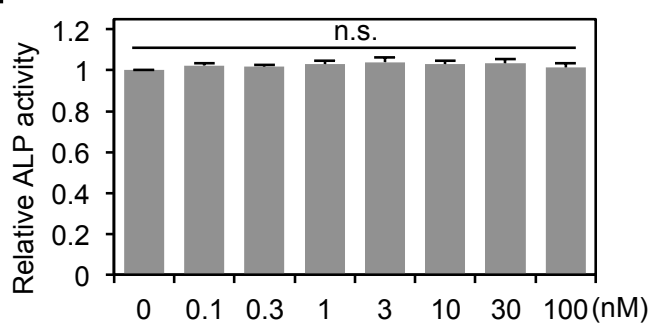

G

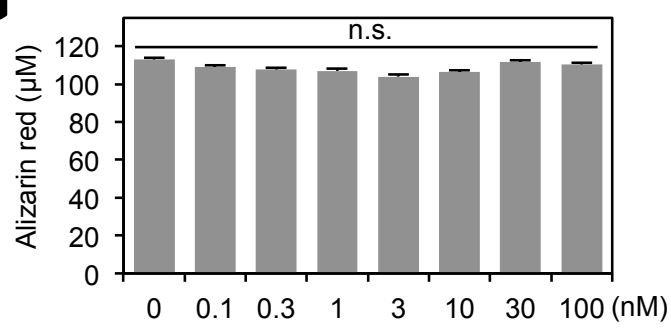


Figure 2
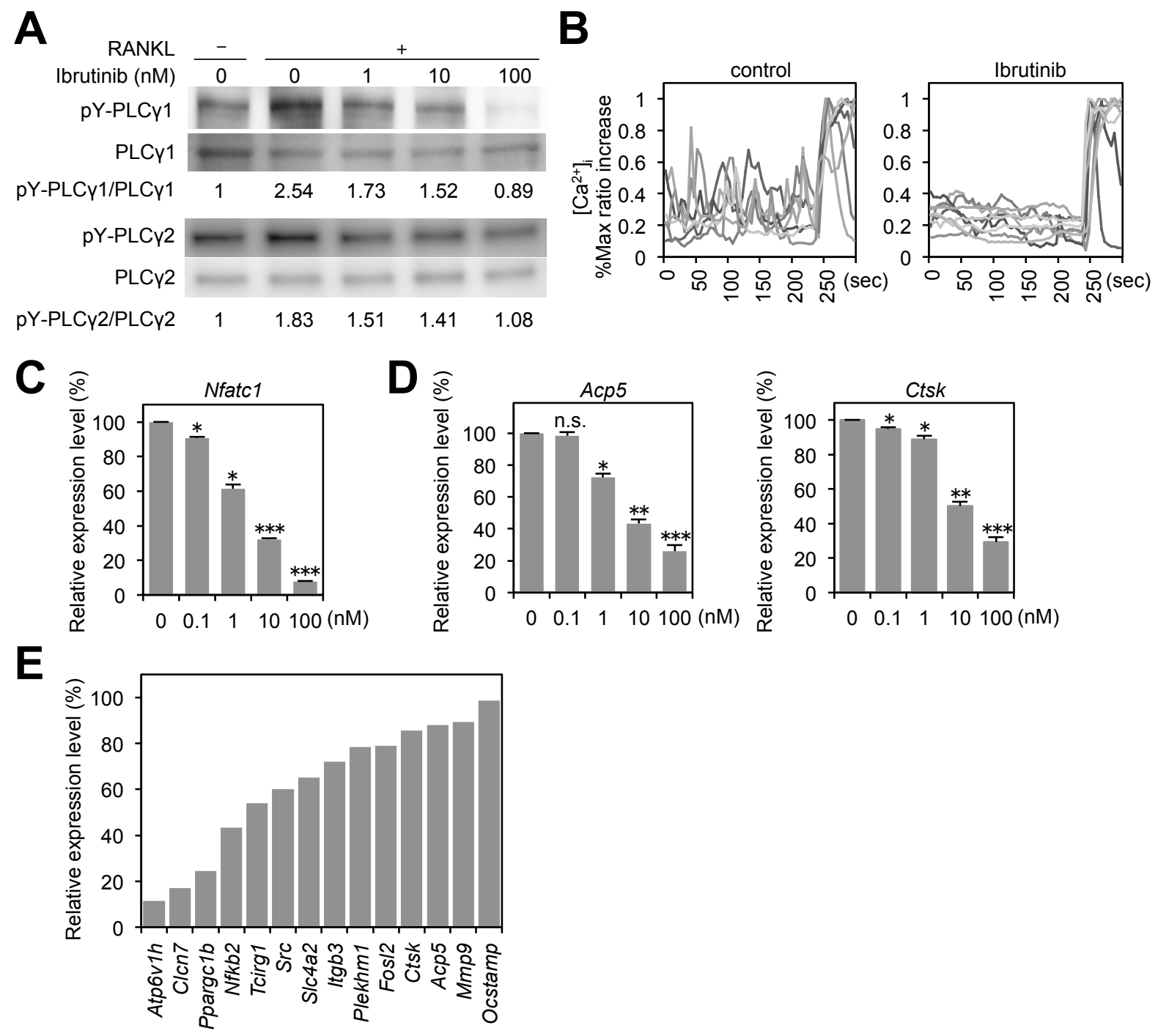
Figure 3
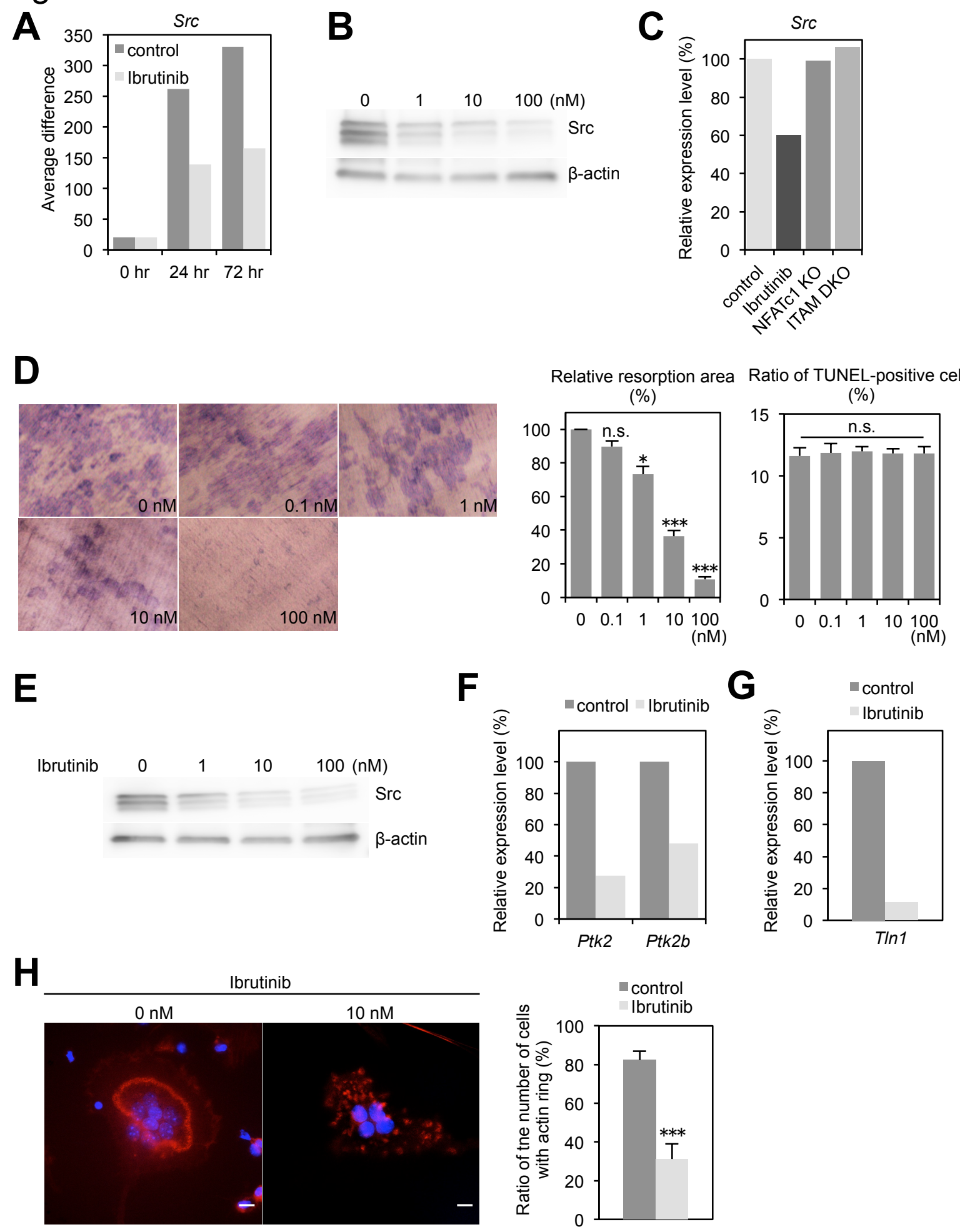


\section{Figure 4}

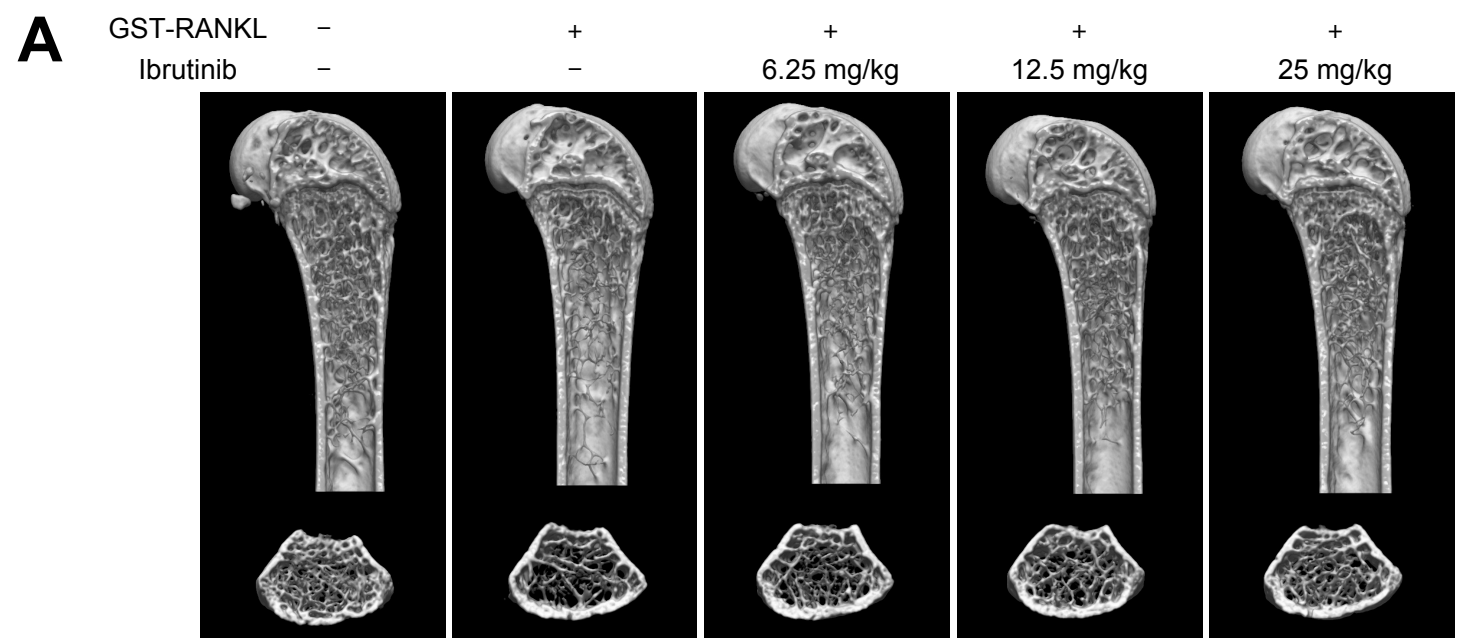

saline $\quad$ RANKL+saline $\quad$ RANKL+ibrutinib $(6.25 \mathrm{mg} / \mathrm{kg}) \quad \mathrm{RANKL}+$ ibrutinib (12.5 mg/kg) RANKL+ibrutinib (25 mg/kg)
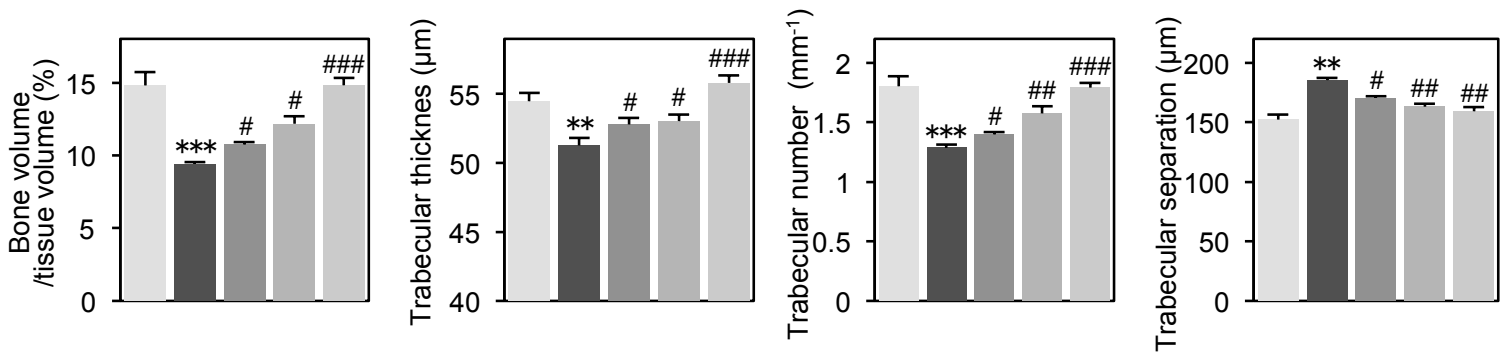

B

saline $\quad \mathrm{RANKL}+$ saline $\quad \mathrm{RANKL}+$ ibrutinib $(6.25 \mathrm{mg} / \mathrm{kg}) \quad \mathrm{RANKL}+\mathrm{ibrutinib}(12.5 \mathrm{mg} / \mathrm{kg}) \square \mathrm{RANKL}+\mathrm{ibrutinib}(25 \mathrm{mg} / \mathrm{kg})$
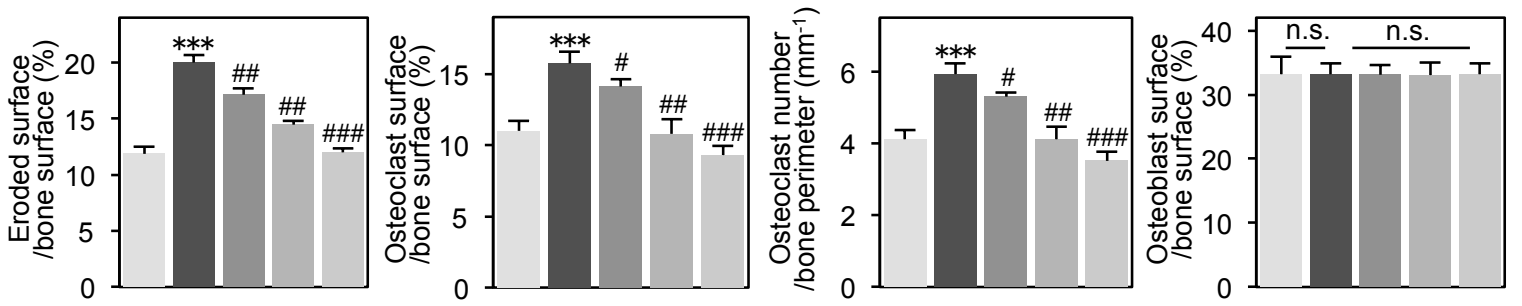

C

saline $\square$ RANKL+saline $\quad$ RANKL+ibrutinib $(6.25 \mathrm{mg} / \mathrm{kg}) \quad \mathrm{RANKL}+\mathrm{ibrutinib}(12.5 \mathrm{mg} / \mathrm{kg}) \quad \mathrm{RANKL}+\mathrm{ibrutinib}(25 \mathrm{mg} / \mathrm{kg})$
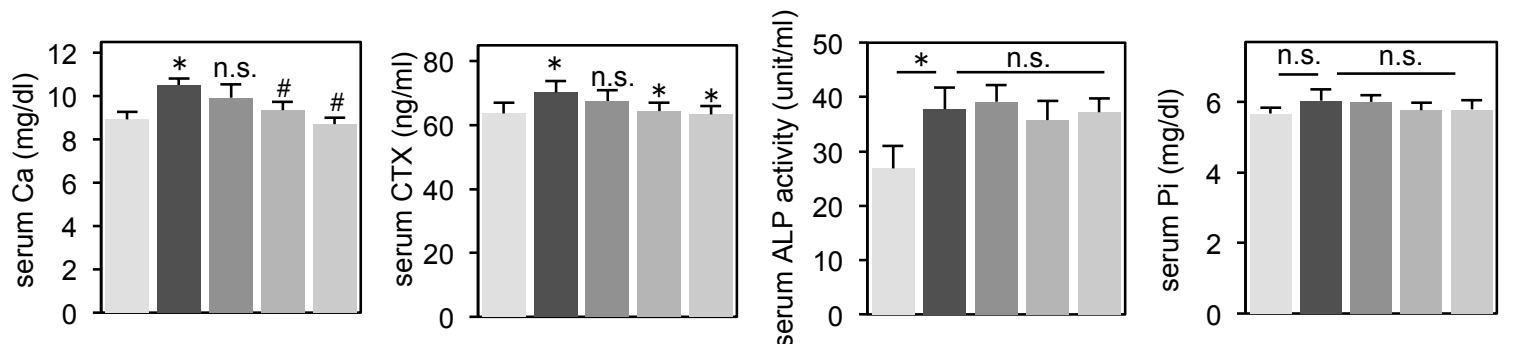